\title{
Tales within Tales and Cutoffs within Cutoffs: What Sets the Mass Scale for Galaxies?
}

\author{
Paul L. Schechter \\ Massachusetts Institute of Technology, Cambridge MA 02139, USA
}

\begin{abstract}
Please answer "yes" or "no":
1. Does the mass function for clusters of galaxies cut off exponentially?

2. Does the luminosity function for galaxies cut off exponentially?

3. Is the dependence of virial velocity on galaxy luminosity a power law?

4. Does the velocity function for galaxies cut off exponentially?
\end{abstract}

\section{Introduction}

The luminosities of cosmic lighthouses are limited by a variety of physical processes. The Eddington limit immediately comes to mind, but like other such limits it is a function of mass. The question of limiting luminosities quickly becomes one of limiting masses.

At least four classes of cosmic lighthouses are on the program for this meeting and each has a different typical mass associated with it. It is my non-expert impression that for both stars and AGN we have some understanding of the physics that limits their masses. I will argue that the mass scale for clusters of galaxies is readily explained, but that our understanding (or at least my understanding) of the mass scale for galaxies is very incomplete.

\section{The Mass Function for Clusters of Galaxies}

The mass scale for clusters of galaxies is set by cosmological parameters which we take to be no different in character than the fundamental microscopic constants. The reigning paradigm is that clusters of galaxies grow by gravitational instability from small density perturbations in an otherwise uniform universe.

We start by asking how large a perturbation is needed at the time of recombination to collapse into an object which has just virialized, and find that we need a density perturbation with a fractional amplitude of roughly 0.001 . We then ask how fluctuations in the underlying dark matter distribution manifest themselves as observed fluctuations in the cosmic microwave background. We find the corresponding fluctuations in the CMB on an angular scale which gives a mass typical of of clusters of galaxies.

The typical mass for clusters of galaxies might manifest itself in many different ways: it might be the mean of a Gaussian, the break in two power laws, a cutoff in a single power law or something else. The typical mass sets the scale 
but it remains to determine the shape of the mass function. For the purpose of the present meeting, we are especially interested the details of its high mass tail.

I did some work on the mass function for clusters as part of my PhD thesis. Gunn and Gott [8] had just done their well known work on the spherical collapse model for clusters. It seemed to me that since we knew that there were galaxies, whatever perturbations gave rise to galaxies would, on a larger scale, give rise to clusters. Starting, for the sake of argument, with the assumption that galaxies were distributed in Poisson fashion I arrived at an expression for the mass function for clusters of galaxies that consisted of a power law with an exponential cutoff.

There were problems with the argument and they had me worried. I had equated two quantities that I knew where not the same thing. The nature of the swindle is more obvious if one describes the quantities in words:

$$
\left(\begin{array}{c}
\text { fraction of } \\
\text { masses } M \\
\text { with } \delta>\delta_{c}
\end{array}\right) \approx\left(\begin{array}{c}
\text { fraction of Universe } \\
\text { collapsed in objects } \\
\text { with masses }>M
\end{array}\right)
$$

This ansatz made it possible to derive a mass function, but I was very nervous about the slope at the low mass end. An argument that gave the same slope in any number of dimensions couldn't possibly be right. I struggled with this for the better part of a year. Some time thereafter Bill Press joined the effort. He generalized my Poisson result to a power-law spectrum of arbitrary index $n$, emphasizing the self-similarity of the process and carried out what, by $\mathrm{Ed}$ Bertschinger's [2] reckoning, appears to have been the first cosmological N-body simulations (with $\mathrm{N}=1000$ ) for the purpose of checking our results. We published what we had 18 and never did find a way to avoid the swindle.

Our mass function,

$$
n(M)=\frac{1}{2 \sqrt{\pi}}\left(1+\frac{n}{3}\right) \frac{\bar{\rho}}{M^{2}}\left(\frac{M}{M *}\right)^{3+n} \exp \left[-\left(\frac{M}{M *}\right)^{(3+n) / 3}\right]
$$

has undergone a number of extensions and improvements. A fudge factor has been successfully explained, and the approach has been "extended" (see Lacey and Cole [11] and the review by Schuecker et al. [20]) to include conditional merger probabilities looking backward and forward in time. It has also been adapted to non-spherical collapse 22]. Its deficiencies have been investigated by N-body experiment, leading several groups to suggest modifications 21,9]. Pierluigi Monaco writes 15]:

The history of the mass function theory is reviewed in Monaco (1998) [14 but it can effectively be summarized in a sentence: there is a simple, effective and wrong way to describe the cosmological mass function. Wrong of course, does not refer to the results but to the whole procedure.

Not only did we get the calculation wrong, we had the input physics wrong as well. We assumed that the matter that was clustering was strictly baryonic. At 
recombination baryonic density perturbations have roughly the same amplitude as the CMB fluctuations, which we now know were much too small to produce today's clusters of galaxies. What we didn't appreciate was that non-baryonic dark matter perturbations, uncoupled to the photons and the baryons, could have been growing while the baryonic perturbations were locked into the photon fluid. Clusters of galaxies are fundamentally self-gravitating, pressure supported spheroids of dark, non-baryonic matter. Their baryons comprise only a small fraction of their mass. And the galaxies from which they take their name include only a small fraction of the baryons.

Such shortcomings notwithstanding, many investigators have found the PressSchechter recipe to be an acceptable first cut at the mass function for clusters of galaxies. The answer to our first question appears to be "yes" - the mass function has an exponential cutoff.

\section{The Luminosity Function for Galaxies}

\section{1 the shape of the luminosity function}

Hubble, Zwicky and George Abell all carried out studies of the luminosity function for galaxies, and and all of them found that there is a characteristic luminosity. They differed considerably, however, on the shape of the luminosity function. Hubble found it to be roughly Gaussian and Abell found the cumulative luminosity function to be a broken power law.

In one of the opening volleys of the science wars, Abraham Maslow wrote [13] wrote:

I suppose it is tempting, if the only tool you have is a hammer, to

treat everything as if it were a nail.

It's true. My hammer was the power law with an exponential cutoff and I hammered on the luminosity function for galaxies 19.

Many investigators have wielded this hammer since, and some have found it unsatisfactory. But it would still seem to be of some use. In their analysis of the SDSS commissioning data, Blanton et al. find, to their evident surprise, that a power law with an exponential cutoff fits their data better than an alternative, non-parametric model [3]. The answer to the second question would also appear to be "yes."

\section{2 implications for the mass function}

From the outset it seemed somewhat inconsistent, perhaps even hypocritical, to use roughly the same functional form for both the mass function for galaxies and the luminosity function for galaxies. One might argue that the same process which gives rise to the cluster mass function gives rise to the galaxy mass function at some earlier time, but this argument is flawed. The idea of self-similar condensation was that the objects seen at one epoch would merge to form larger objects. How could they merge and yet survive? We would be eating our cake and 
having it too. If the same process were to work twice, an early epoch of structure had to be frozen in such a way that it could survive subsequent mergers. This question has been addressed repeatedly, both theoretically and experimentally with N-body simulations. Only recently have the latter begun to give reliable answers. We discuss them in greater detail below, but for the moment the important conclusion is that a small but significant fraction of the mass, perhaps $10 \%$, survives in what look like galaxies.

\section{The Faber-Jackson and Tully-Fisher Relations}

In papers published within a year of each other, Faber and Jackson [7] (henceforth FJ) and Tully and Fisher [25] (henceforth TF) found, respectively, that elliptical and spiral galaxies exhibited a similar scaling between internal velocities (velocity dispersion and circular velocity) and galaxy luminosity. They found luminosity varied roughly as the fourth power of observed velocity, with a scatter about the mean relation of $30-40 \%$. The FJ relation has since been refined to include surface brightness by Dressler et al. [6] and Djorgovski and Davis [5] giving what the latter call the "fundamental plane."

Bernardi et al. [1] recently completed a massive study of the fundamental plane finding, among other things, that it is, very nearly, a plane. When they compute the mean velocity dispersion at fixed absolute magnitude and correct for evolution they see little if any deviation from a power law - the FJ relation holds. The answer to our third question is another "yes".

\section{The Velocity Function for Galaxies}

\section{1 why not the mass function?}

As our subject is the mass scale for galaxies, the distribution of virial velocities - the "velocity function" - may seem a diversion, deflecting us from our goal. Alas it is nearly impossible to measure masses for galaxies. Mass measurements combine a velocity and a scale length measurement. While one can measure scale lengths for the baryons one sees in galaxies - stars or gas - there is every reason to believe that this is not representative of the dark matter. Baryonic collapse is dissipational while dark matter is non-dissipational. Differential collapse by roughly a factor of ten is not unreasonable. We exhaust the supply of stars and gas with which to measure galaxy measure rotation curves and velocity dispersions before we reach the outer limits of the dark matter.

By contrast, the velocities we measure in the baryonic matter are representative of the dark matter. The virial theorem, in its simplest incarnation, tells us that the time averaged squared (virial) velocity is given by

$$
\left.<v^{2}\right\rangle=\left\langle\vec{r} \cdot \frac{\partial \Phi}{\partial \vec{r}}\right\rangle
$$


where $\Phi$ is the gravitational potential. The dark matter and the stars orbit in the same potential, and would have the same virial velocity on the same orbit. Since galaxies appear to be nearly isothermal, the righthand side of the above equation is not a strong function of position, and it is not unreasonable to take the virial velocity for the baryonic matter as a proxy for that of the dark matter.

A similar circumstance holds for studies of clusters of galaxies, which sometimes use "temperature functions" rather than mass functions. Masses for clusters are similarly dependent upon details of how the scale length is measured. By contrast measurement of the temperature of X-ray emitting gas is relatively straightforward.

\section{2 the observations}

At first it would seem a straightforward matter to compute a "velocity function" from the luminosity function and either the FJ or TF relation, especially if the luminosity-velocity relations were indeed power laws. For example, if if the luminosity function cut off as $\exp (-L / L *)$ and luminosity varied as $v^{4}$, one would have the velocity function cutting off as $\exp \left[-(v / v *)^{4}\right]$. If the luminosity-velocity relation saturated at large velocities, the cutoff would be sharper.

But there are complications. Bernardi et al. [1] point out that in carrying out the above calculation, one must take into account the spread in virial velocity at fixed luminosity. Moreover, there is a potential problem [10] in covariances of the luminosity function and the velocity-luminosity relation with hidden variables. Pahre et al. (as reported by Kochanek [10]) have taken such covariances into account. Their velocity function, as shown in Kochanek's figures 6,7 and 9, cuts off exceedingly sharply.

The separations of multiply imaged quasars vary as the square of the virial velocity. Kochanek [10] has computed a velocity function from these, and while the statistics are meager, it is consistent with an exponential cutoff. Taking all of this into consideration, the answer to our fourth question, as determined from observations, is "most probably."

\section{3 the simulations}

Determining the merger histories for present day galaxies from observations is a daunting problem, even for our own Milky Way. Luckily the hierarchical condensation of dark matter can be simulated with N-body experiments, at least in principal. But for many years N-body practitioners were frustrated by their simulations - they formed beautiful clusters but there were no galaxies. Previous generations of structure merged to form the present generation. Sometime around 1986 this phenomenon was given a name by Jerry Ostriker - overmerging. In the last two or three years several groups (notably those based in New Mexico, Seattle and Garching) appear to have traced the overmerging problem to the numerical necessity of softening the $1 / r$ gravitational potential. It now seems that substructure can survive to the present epoch. 
This is a major accomplishment, and cause for celebration, but we shouldn't forget that while some substructure survives, most of it is destroyed 24]. The astronomical jargon used to describe this is still in flux, but the substructures which survive (identified with today's galaxies) are called sub-halos and the larger structures in which they are embedded (identified with today's clusters) are called halos. To order of magnitude, it appears that $10 \%$ of the dark matter remains more closely associated with sub-halos rather than the larger halos. I

The next step after identifying sub-halos is to construct a mass function. Some investigators choose to construct a velocity function as well, either for the sake of comparison with observation or because it is less sensitive to the details of the algorithm used to identify sub-halos.

While the results are relatively new and the details may change as the experiments improve, two important conclusions can already be drawn. First, it appears that the mass function for sub-halos is very much steeper than the luminosity function for galaxies. Second, there is at best weak evidence for an exponential cutoff in the masses of sub-halos - the mass functions look very much like power laws.

The same holds true for velocity functions. They look like power laws without any obvious cutoff [4. The answer to our fourth question, as determined from N-body simulations, is "evidently not." In an odd way this is reassuring - it is not obvious what would introduce either a mass scale or velocity scale for the sub-halos, so it is just as well that we don't see one.

We face a serious dilemma. Observation gives a positive answer to our fourth question and N-body experiment gives a negative answer.

\section{The Baryonic Mass Function for Galaxies}

As discussed in the previous section, the dark matter mass function for galaxies is perversely difficult to measure. By comparison the baryonic mass function is fairly easy - indeed to first order it is the luminosity function scaled by a mass-to-light ratio.

The N-body experiments in which sub-halos form within halos involve only dark matter particles - they interact only by non-dissipative, mutual gravita-

$\overline{1}$ This raises an interesting question question of physics which, while not strictly applicable to the universe in which we live, may nonetheless help to understand it. The paper by Bill Press and myself made much of the self-similarity of the growth of structure. This depended first, upon the power-law nature of the perturbation spectrum and second, on the assumption of an Einstein-de Sitter cosmology. Today we believe that the perturbation spectrum at recombination is the product of a number of competing processes that would have destroyed the power-law nature of any input spectrum. But we can still ask what would happen in a hypothetical Einstein-de Sitter universe in which the input spectrum was a power law. Would selfsimilarity still hold? In particular, would the fraction of mass in sub-halos always be the same percentage of the total mass at all times? Or would we expect to find sub-sub-halos inside sub-halos and so forth? I suspect that this is the case, although I doubt anyone has the code or the perseverance to carry this out. 
tion. By contrast, luminosity functions, both optical (for galaxies) and X-ray (for clusters), describe baryonic matter, which can radiate away its energy. All manner of physics can cause dark matter and baryonic matter, however uniformly distributed at first, to become separated over the course of time. There is every reason to think that mass function for baryons will therefore be quite different from the mass function for sub-halos (and halos).

A variety of "gastrophysical" mechanisms have been invoked to explain the differences between the observed (baryonic) luminosity function for galaxies and sub-halo mass function derived from N-body experiments. At the low mass end, baryons must either be ejected from sub-halos or something must prevent them from collapsing along with the collapse of dark matter. Somerville 23 has used the word "squelching" to describe the suppression of dwarf galaxy formation by the re-ionization of the IGM. There may be some disagreement about the physics but it's an apt word to describe the phenomenon.

Whatever causes the flattening of the mass function at the low mass end, something else must cause a steepening at the high mass end. White and Rees 27 proposed that the cooling time for baryonic gas, assumed to be uniformly distributed in a dark matter potential well, increases with increasing mass to the point at which the baryons no longer have time to cool and condense. It should be remembered, however, that White and Rees went to considerable lengths to emphasize that substructure would be destroyed. If substructure is not destroyed, then the gas associated with this substructure would be clumpy and would have much shorter cooling times.

Whatever the detailed physics, the underlying idea of White and Rees would seem to be that sub-halos with large internal velocities exist, but we don't see them because their baryons could not and have not condensed.

A half-dozen groups are engaged in a heroic enterprise that goes by the name "semi-analytic modeling." They attempt to form galaxies in dark matter halos, taking care to include the gravitational condensation of halos and the gas-dynamical condensation of baryons within these halos. They include star formation, energy feedback, chemical enrichment and obscuration by dust. All of these are accomplished by a series of more-or-less realistic recipes. More recently some of these modelers have substituted high resolution N-body simulations for analytically derived halo histories. These models have many free parameters - enough to fit almost any set of constraints. Yet with all these parameters, the modelers still have difficulty in cutting off the bright end of the luminosity function. The adopted values of the parameters seem extreme, and in some cases the assumptions seem ad-hoc.

The TF and FJ relations place strong constraints on the recipes. One sort of recipe might have the fraction of the baryons which condense within a halo decrease as the halo velocity dispersion increases. A second sort might have the condensation of baryons in a halo be an all-or-nothing affair, but with baryon condensation increasingly unlikely with increasing halo dispersion. Recipes of the first sort might run afoul of the velocity-luminosity constraints, producing halos with large velocities but relatively low luminosities. 
I suspect that something on the order of the solution proposed by White and Rees will ultimately prove successful in inhibiting the collapse of baryons into the largest halos, thereby producing a baryonic mass cutoff for galaxies. But for the moment the modelers have not yet persuaded each other; one wonders whether they have even persuaded themselves.

For the sake of argument let's grant that something squelches the condensation of baryons into low mass halos that something else inhibits the condensation of baryons into high mass halos. This brings us back to the velocity function constructed from gravitational lensing. If baryonic infall had no effect on the structure of sub-halos, the distribution of lens separations could be derived from the velocity function determined from N-body simulations and one ought not to see a cutoff. But White and Kochanek [26] have argued that baryonic infall does alter the gravitational potentials of halos, particularly at the relatively small radii sampled by lensed quasars, so even the lensed quasar separation distribution may exhibit a cutoff, or at least a kink. While this might solve our problem, it should be noted (as they do) that their model reproduces the observed distribution of lens separations only if the baryonic mass fraction in galaxies is larger than is usually assumed.

\section{6 cD Galaxies: Sub-Halos or Halos?}

No discussion of galaxies as lighthouses would be complete without mention of cD galaxies. It is helpful to make a distinction between those which are instantaneously the brightest and those which are the brightest when averaged over cosmic timescales. In their contributions to these proceedings LONGAIR and CESARsky discuss sub-mm sources and ULIRGs, which are instantaneously the brightest galaxies in the universe. Averaged over time this honor may belong, instead, to the $\mathrm{cD}$ galaxies found at the centers of many (but not all) clusters of galaxies.

The "cD" classification originated in a paper by Matthews, Morgan and Schmidt 12] (henceforth MMS). The lowercase "c" comes from Morgan's classification for supergiant stars and the uppercase D stands for diffuse. With NGC 6166 in Abell 2199 as the archetype [16], these galaxies had large, low surface brightness halos. They frequently seemed to harbor radio sources.

The designation $\mathrm{cD}$ is sometimes taken to stand for the words central and dominant. While this was not the original meaning of MMS, there is more than a bit of truth in it. Such galaxies are indeed found preferentially at the centers of clusters of galaxies and they tend to be the dominant galaxy. Oegerle and Hill 177 have found that they also tend to lie at the center of the velocity distribution for the cluster in which they reside.

There is an old but still interesting question as to whether the $\mathrm{cD}$ galaxies are members of the same population as the other galaxies in a cluster, or are in some way special. If the luminosities of galaxies really do cut off exponentially, there's no explaining cD's. 
There are other peculiarities associated with cD's as well - their velocity dispersion profiles rise rather than fall as a function of radius. This is consistent with their extended envelopes and even with a flattening of the slopes of their light profiles. Moreover they seem to have an excess of globular clusters.

The same N-body experiments which give rise to interesting substructure also seem to produce a condensation at the center of each cluster which has the density of a galaxy. The special position of galaxies at the centers of cluster potentials might make them different. Today they seem largely to be star piles, the piling up of fragments of previously formed galaxies.

At earlier times cD's may have been special in a different way. The galaxies at the centers of potentials are the natural sinks for cooling baryons, and one expects baryons to collect there - one would think in disks. In the semi-analytic models the galaxy at the center of a halo occupies a unique position - it is the only galaxy which accretes baryons. In such a model a dark matter halo collects baryons only until it is subsumed into a larger halo in which it is no longer in the privileged central position. The other galaxies in the halo are called satellites to distinguish them from the central galaxy. But if they have any baryons, then at one time they must have been at the center of the cluster. In short, every galaxy must have at one time been a central dominant galaxy, though not a cD galaxy in the sense of MMS.

\section{Conclusion}

The answers to the four questions posed in the abstract are yes, yes, yes and yes-and-no. The observed velocity function for galaxies appears to cut off exponentially while the velocity function for N-body sub-halos appears not to. There is as yet no detailed physical model which cuts off the condensation of baryons into dark matter halos in a manner which conforms to the observations.

\section{References}

1. Bernardi, M. et al. 2001 astro-ph/0110344)

2. Bertschinger, E. 1998, ARAA, 36, 599

3. Blanton, M. R. et al. 2001, AJ, 121, 2358

4. Bullock, J. S., Kravtsov, A. V., \& Weinberg, D. H. 2001, ApJ, 548, 33

5. Djorgovski, S. \& Davis, M. 1987, ApJ, 313, 59

6. Dressler, A., Lynden-Bell, D., Burstein, D., Davies, R. L., Faber, S. M., Terlevich, R., \& Wegner, G. 1987, ApJ, 313, 42

7. Faber, S. M. \& Jackson, R. E. 1976, ApJ, 204, 668

8. Gunn, J. E. \& Gott, J. R. I. 1972, ApJ, 176, 1

9. Jenkins, A., Frenk, C. S., White, S. D. M., Colberg, J. M., Cole, S., Evrard, A. E., Couchman, H. M. P., \& Yoshida, N. 2001, MNRAS, 321, 372

10. Kochanek, C. S. 2001, in The Dark Universe meeting at STScI, April 2-5, 2001

M. Livio, ed., Cambridge University Press, (astro-ph/0108160)

11. Lacey, C. \& Cole, S. 1993, MNRAS, 262, 627

12. Matthews, T. A., Morgan, W. W., \& Schmidt, M. 1964, ApJ, 140, 35 
13. Maslow, A. H. 1966, The Psychology of Science; A Reconnaissance (New York: Harper, Row \& Publishers), p. 15

14. Monaco, P. 1998, Fundamentals of Cosmic Physics, 19, 157

15. Monaco, P. 1998, ASP Conf. Ser. 146: The Young Universe: Galaxy Formation and Evolution at Intermediate and High Redshift, 318

16. Morgan, W. W. \& Rountree Lesh, J. 1965, ApJ, 142, 1364

17. Oegerle, W. R. \& Hill, J. M. 1994, AJ, 107, 857

18. Press, W. H. \& Schechter, P. 1974, ApJ, 193, 437

19. Schechter, P. 1976, ApJ, 203, 297

20. Schuecker, P., Böhringer, H., Arzner, K., \& Reiprich, T. H. 2001, A\&A, 370, 715

21. Sheth, R. K. \& Tormen, G. 1999, MNRAS, 308, 119

22. Sheth, R. K., Mo, H. J., \& Tormen, G. 2001, MNRAS, 323, 1

23. Somerville, R. S. 2001 (astro-ph/017507)

24. Springel, V., White, S. D. M., Tormen, G., \& Kauffmann, G. 2001, MNRAS, 328, 726

25. Tully, R. B. \& Fisher, J. R. 1977, A\&A, 54, 661

26. White, M. \& Kochanek, C. S. 2001, ApJ, 560, 539

27. White, S. D. M. \& Rees, M. J. 1978, MNRAS, 183, 341 